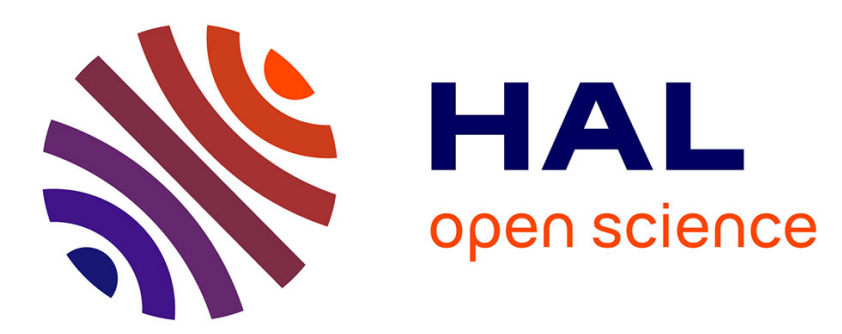

\title{
Ionic aggregation-induced emission: bulky hydrophobic counterions light up dyes in polymeric nanoparticles
}

\author{
Ilya O Aparin, Nina Melnychuk, Andrey S Klymchenko
}

\section{To cite this version:}

Ilya O Aparin, Nina Melnychuk, Andrey S Klymchenko. Ionic aggregation-induced emission: bulky hydrophobic counterions light up dyes in polymeric nanoparticles. Advanced Optical Materials, 2020, 8 (14), pp.2000027. 10.1002/adom.202000027 . hal-03020565

\section{HAL Id: hal-03020565 https://hal.science/hal-03020565}

Submitted on 23 Nov 2020

HAL is a multi-disciplinary open access archive for the deposit and dissemination of scientific research documents, whether they are published or not. The documents may come from teaching and research institutions in France or abroad, or from public or private research centers.
L'archive ouverte pluridisciplinaire HAL, est destinée au dépôt et à la diffusion de documents scientifiques de niveau recherche, publiés ou non, émanant des établissements d'enseignement et de recherche français ou étrangers, des laboratoires publics ou privés. 
DOI: $10.1002 /(($ please add manuscript number))

Article type: (Full Paper)

Ionic aggregation-induced emission: bulky hydrophobic counterions light up dyes in polymeric nanoparticles

Ilya O. Aparin, Nina Melnychuk, and Andrey S. Klymchenko*

Laboratoire de Bioimagerie et Pathologies, UMR 7021 CNRS, Université de Strasbourg, 74 route du Rhin, 67401, Illkirch, France

Dr. I. O. Aparin, Dr. N. Melnychuk, Dr. A. S. Klymchenko

Laboratoire de Bioimagerie et Pathologies, UMR 7021 CNRS, Université de Strasbourg, 74 route du Rhin, 67401, Illkirch, France

Corresponding author: Dr. A.S. Klymchenko

E-mail: andrey.klymchenko@unistra.fr

Keywords: dye-loaded polymeric nanoparticles, aggregation-induced emission, aggregationcaused quenching, bulky hydrophobic counterions, single-particle microscopy

\begin{abstract}
Dye-loaded polymeric nanoparticles emerge as powerful bioimaging tools, but their assembly is challenged by aggregation-caused quenching (ACQ) of flat fluorophores. Aggregationinduced emission (AIE) proposes an effective solution against ACQ by exploiting propellershaped neutral fluorophores without polymeric blending. Fighting ACQ of ionic dyes in polymeric nanoparticles can be achieved by bulky hydrophobic counterions. Here, we aim to generate an AIE phenomenon in poorly emissive ionic dyes using bulky counterions. Three cationic hemicyanine dyes of styryl pyridinium family have been synthesized: two planar fluorophores featuring ACQ and one propeller-shaped AIE fluorophore. We found that in water bulky fluorinated tetraphenylborates can light up all three dyes, including planar nonAIE fluorophores. The described "ionic" AIE (iAIE) with bulky counterions enables preparation of 50-nm dye-loaded polymeric NPs showing $40 \%$ quantum yield at $500 \mathrm{mM}$ dye loading, whereas small anions favor poorly emissive large aggregates. Single-particle microscopy reveals that these NPs emit without blinking 50-fold brighter than quantum dots605 (at 470-nm excitation). iAIE opens the route to assembling charged intrinsically non-AIE fluorophores into bright fluorescent (nano)materials.
\end{abstract}




\section{Introduction}

The surge of fluorescence bioimaging technics encourages the research on new bright dyes and materials with improved properties and fluorescent signal amplification techniques. Fluorescent nanoparticles of small size have been proved to be a promising tool for bioimaging as they largely surpass organic dyes in terms of brightness. ${ }^{[1-4]}$ Quantum dots, ${ }^{[5-7]}$ dye-doped silica nanoparticles, ${ }^{[8,9]}$ nanodiamonds, ${ }^{[10]}$ carbon dots ${ }^{[11]}$ have been intensively studied as bioimaging agents in the last decade. Fluorescent organic nanoparticles assembled from organic dyes is an attractive alternative because they can address the existing challenges in the field, such as poor biodegradability, potential long-term toxicity, limited brightness, etc. $^{[12]}$ In this respect, dye-loaded polymeric nanoparticles are particularly powerful, ${ }^{[13]}$ because polymeric matrix can ensure stability of the nanoparticles preventing organic dyes from bleaching in biological media, while polymer design allows preparation of small NPs with size control in a broad range from 7 till $200 \mathrm{~nm} \cdot{ }^{[14,15]}$ However, when the dyes are confined within a nanostructure at very high local concentration, they tend to pi-stack into $\mathrm{H}$ aggregates, ${ }^{[16-19]}$ leading to so-called aggregation-caused quenching (ACQ).

A number of approaches have been developed to fight ACQ in optical materials. ${ }^{[4]}$ The classical approach is to incorporate bulky groups into the fluorophore, which would prevent the dyes form pi-stacking. ${ }^{[20-24]}$ However, this approach requires tedious multi-step synthesis of corresponding dyes and ACQ in these systems is usually not completely prevented. ${ }^{[23]}$ Probably the most popular approach to fight ACQ is aggregation-induced emission (AIE), originally proposed by Tang and co-workers. ${ }^{[25]}$ In this case, the fluorophore generally presents twisted propeller-shaped topology that blocks $\mathrm{H}$-aggregation favoring formation in the solid-state highly emissive aggregates. ${ }^{[26-29]}$ The effect is further amplified by poor emission of AIE fluorophores (AIEgens) in organic solvents, because their twisted topology favors excitation energy losses by molecular rotation, usually through twisted intramolecular charge transfer (TICT) ${ }^{[30]}$ In the solid state, this rotation is prohibited (so-called restriction of intramolecular rotation, RIR), leading to enhancement of fluorescence emission in the dye aggregates. ${ }^{[26-28]}$ AIE expanded to different fields of optical materials, including organic lightemitting diodes, ${ }^{[31]}$ liquid-crystal displays, ${ }^{[32]}$ light-harvesting, ${ }^{[33,34]}$ short-wave nearinfrared, ${ }^{[35]}$ and photo-switching ${ }^{[36]}$ materials, solar concentrators, ${ }^{[37]}$ sensors, ${ }^{[38]}$ theranostics agents, ${ }^{[27]}$ etc, and it significantly impacted development of dye-based nanoparticles. ${ }^{[39-44]}$ In particular, AIE enabled preparation of bright fluorescent polymeric nanoparticles, either by direct polymerization of AIE monomers ${ }^{[43,44]}$ or in core-shell systems with the core formed by AIEgen and the shell made of a polymeric biocompatible material. ${ }^{[3,35,45-47]}$ However, preparation of dye-loaded polymeric NPs using AIE dyes meets some challenges, because dispersion of AIEgens within the polymer may lead to dye quenching because its unique crystalline organization is disrupted by the polymer. ${ }^{[48]}$ Moreover, AIEgens are dominated by neutral molecules, whereas the examples of intrinsically charged AIEgens are very rare. ${ }^{[49,50]}$

Charged fluorophores, bearing cationic (or anionic) groups involved in the electronic conjugation, such as cyanines and rhodamines, ${ }^{[51]}$ are particularly attractive because they are characterized by strong electronic delocalization leading to fluorophores of high extinction coefficient and fluorescence quantum yield. However, these dyes are among the most prone to ACQ because they are extended pi-conjugated structures. ${ }^{[16-19]}$ Moreover, their small inorganic counterions cannot prevent dye-dye pi-stacking, probably because of strong 
solvation (hydration) and small size of these counterions, as well as dissociative behavior of dye $^{+} / a^{-}$ion ${ }^{-}$pair. These properties do not allow the small inorganic counterions to separate charged fluorophores within the aggregates, and, therefore, the dyes tend to form strongly quenched H-aggregates. ${ }^{[16]}$ In the early studies the groups of $\mathrm{Yao}^{[52,53]}$ and Warner ${ }^{[54,55]}$ described the possibility to minimize ACQ in these charged dyes using bulky hydrophobic counterions, based on tetraphenylborate (TPB) and bistriflimide families. These approaches have been applied to variety of fluorophores and counterions. ${ }^{[56,57]}$ However, the NPs of pure $\mathrm{dye}^{+} /$counterion $^{-}$salts are not stable in biological environment, because they tend to dissolve in hydrophobic compartments of cells showing leakage of the dyes. ${ }^{[57,58]}$ In 2014, we introduced a concept for preparation of dye-loaded polymeric NPs using ion pairs of cationic rhodamine dye (R18) with bulky hydrophobic counterions. ${ }^{[59]}$ Bulky TPBs, especially their perfluorinated analogues (F5-TPB and F12-TPB, Fig. 1), are able to prevent ACQ in polymeric nanoparticles, ${ }^{[57,59,60]}$ because these anions serve as spacers between the cationic dyes and thus prevent their pi-stacking. On the other hand, bulky hydrophobic counterions are essential for encapsulation of the dyes inside the polymer matrix without further leakage into the biological medium. ${ }^{[60]}$ The concept has been extended to cyanines, which enables controlling NPs color allowing multicolor long term tracking of living cells. ${ }^{[61]}$ It has been also found compatible with different types of counterions, including different fluorinated TPBs, tetrakis(trifluoromethyl)borate, aluminium complex and borohydrides, ${ }^{[60,62]}$ showing that the concept of bulky counterion is rather universal and not limited to one type of anion. Importantly, bulky counterions due to ionic forces also brings the dyes together inside the polymer matrix, which leads to fast dye-dye communication by excitation energy migration. ${ }^{[59,63]}$ The latter results in giant light-harvesting phenomenon, where 10,000 dyes can transfer energy to a single energy acceptor. ${ }^{[64]}$ This nanoantenna has already been applied for single molecule detection at ambient sunlight conditions ${ }^{[64]}$ and for the development of ultrabright nanosensors for amplified detection of nucleic acids. ${ }^{[65]}$

So far, it remains unclear whether this counterion approach can be applied to poorly emissive ionic dyes, in order to produce the AIE phenomenon. Recently, we showed that counterions can improve emission properties of AIEgens, especially in the dye-loaded polymeric NPs. ${ }^{[50]}$ In this respect, the current challenge is to apply this bulky counterion approach to produce AIE phenomenon for dyes that intrinsically do not exhibit AIE behavior. In literature, several types of dyes, poorly emissive in organic solvents, have been shown to enhance their emission after association with bulky anions in aqueous media. ${ }^{[52,53,56]}$ However, these systems showed limited fluorescence quantum yields and they have not been really applied for preparation of stable dye-loaded polymeric NPs.

As a promising family of cationic dyes that are poorly emissive in organic solvents without clear AIE characteristics, we selected styryl pyridinium (SP) dyes. Their strongly dipolar nature and flexible conjugation makes them poorly emissive in organic solvents, being highly sensitive to the environmental properties, such as polarity and viscosity. ${ }^{[66-69]}$ Moreover, lipophilic analogues of SP dyes are practically non-fluorescent in water because of strong ACQ, without showing AIE character. Their fluorogenic (light up) response to viscous environments has been extensively used to design fluorescent probes for biological membranes. ${ }^{[68-71]}$ A confinement of an SP dye within anionic metal-organic framework have also led to fluorescence light up. ${ }^{[72]}$ However, only one rare report have shown fluorescence enhancement of an SP dye in water due to a bulky anion effect. ${ }^{[73]}$ So far, no attempts have 
been made to tune AIE properties of SP dyes and they have not been used to fabricate stable polymeric NPs. Thus, SP dyes are promising unexplored dye family for testing our hypothesis.

In the present work, we synthesized three new SP dyes having controlled ACQ/AIE profile: two planar dyes and a propeller shaped molecule, featuring modulated ACQ and AIE characteristics. We found that bulky hydrophobic counterions based on perfluorinated TPB induced drastic fluorescence light up in water. The latter was observed for all SP dyes, including planar derivatives without AIE characteristics. Thus, AIE phenomenon can be observed for non-AIE dyes purely due to counterion effect. This ionic aggregation-induced emission (iAIE) has been applied for preparation of bright dye-loaded polymeric NPs, featuring high fluorescence brightness (50-fold brighter than QDdot-605) and good photostability without detectable blinking. The observed iAIE phenomenon provides a bridge between two complementary concepts of fighting ACQ, AIE and bulky counterions, and opens the route to assembling charged intrinsically non-AIE fluorophores into bright fluorescent (nano)materials.

\section{Results and discussion}

\subsection{Design, synthesis and fluorescent properties of SP dyes}

We designed three SP dyes bearing diethylamino, diphenylamino and carbazolyl auxochrome groups: EtSP18, PhSP18 and CzSP18, correspondingly (Fig. 1a). Octadecyl chain was introduced to increase hydrophobicity of the dyes to favor their aggregation and encapsulation into polymeric NPs. ${ }^{[60,74]}$ EtSP18 dye has a planar chromophore structure capable to dissipate excitation energy with rotation of diethylamino moiety and phototoisomerisation. Due to strong polarizability of EtSP18, typical for hemicyanine dyes, it is expected to undergo strong dipole-dipole interaction and aggregation-caused quenching in solid state. To render this dye some AIE character, diethylamino group was replaced with a propeller-shaped diphenylamine moiety (PhSP18), which is extensively used in AIEgens. ${ }^{[27,28]}$ Then to block the AIE property, we designed CzSP18, where we replaced diphenylamine moiety with carbazolyl one rendering the dye perfectly planar and thus bringing back ACQ through pi-stacking. Comparison of CzSP18 and PhSP18 would allow us to evaluate the role of AIE character of the dye in the counterion-controlled solid-state emission.

SP dyes PhSP18, EtSP18, CzSP18 were synthesized by adopting previously developed methodology (Fig. 1a). ${ }^{[75]}$ Briefly, 4-picoline was quaternized with octadecyl iodide in acetonitrile under reflux with excellent yield of the picolinium salt. Knoevenagel reaction of $\mathrm{N}$-octadecyl picolinium iodide with 4-( $N, N$-diphenyl)benzaldehyde, 4-(9carbazolyl)benzaldehyde or 4-(N,N-diethylamino)bezaldehyde was performed in boiling acetonitrile with piperidine as a catalyst, yielding PhSP18, CzSP18, EtSP18, respectively. The obtained dyes were recrystallized either from absolute ethanol or acetonitrile to obtain pure crystalline compounds. All the compounds in form of iodide [ $\left.\mathbf{I}^{-}\right]$salts were characterized with 1H, 13C NMR and HRMS. Then, using simple two-phase ion exchange followed by flash-chromatography purification, we obtained individual ion pairs of the cationic dyes with anions that drastically differ by their diameter: (i) small inorganic anions, perchlorate [ClO ${ }_{4}^{-}$] $(\sim 0.33 \mathrm{~nm})$ and hexafluorophosphate $\left[\mathbf{P F}_{6}^{-}\right](\sim 0.35 \mathrm{~nm})$ and (ii) bulky hydrophobic anions, 
tetraphenylborate [TPB] $(\sim 1.08 \mathrm{~nm})$, tetrakis(pentafluorophenyl)borate [F5-TPB] $(\sim 1.16 \mathrm{~nm})$ and tetrakis[3,5-bis-(1,1,1,3,3,3-hexafluoro-2-methoxy-2-propyl) phenyl]borate [F12-TPB] $(\sim 1.6 \mathrm{~nm})$ (Fig. 1b).

a

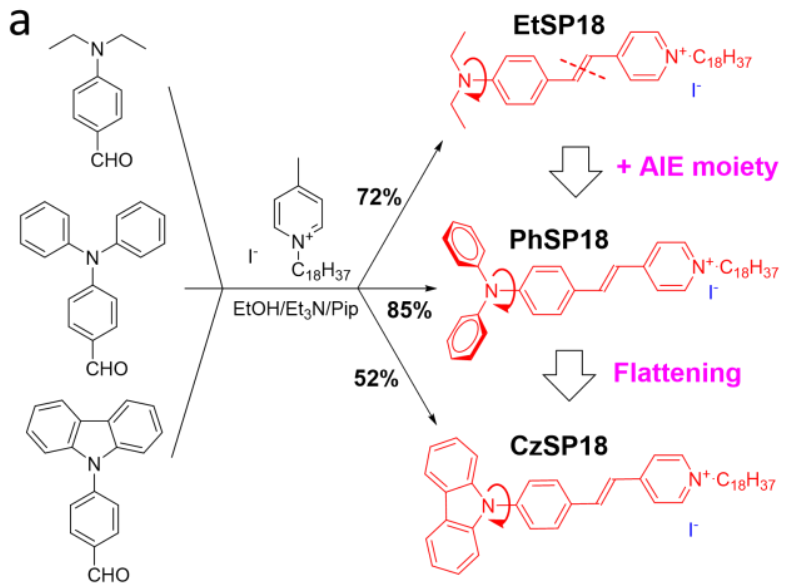

b

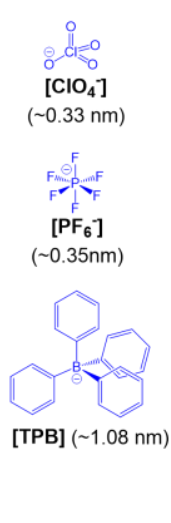

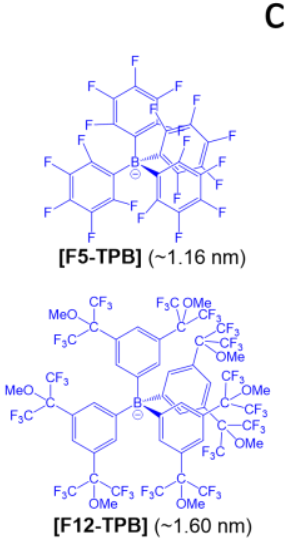

C Ion association NPs

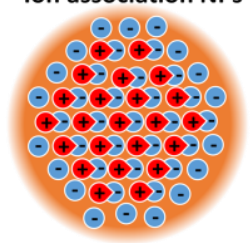

Polymeric NPs

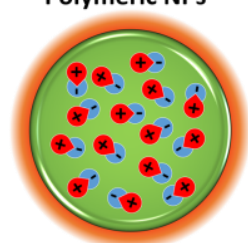

Figure 1. Design of SP dyes and nanoparticles. (a) Synthesis of EtSP18, PhSP18 and CzSP18 dyes; (b) Counterions explored in this study; (c) Schematic representation of pure dye NPs and dye-loaded polymeric NPs.

Next, we studied photophysical properties of EtSP18, PhSP18 and CzSP18 dyes as iodide salts in organic solvents of low and high viscosity: acetonitrile and ethylene glycol, respectively. In the following sequence, CzSP18 $\rightarrow$ PhSP18 $\rightarrow$ EtSP18, absorption maximum of the dyes in both solvents shifted to the longer wavelengths, which was accompanied by an increase in the extinction coefficient. These bathochromic shifts reflect the increase in the electron donor ability of the aniline nitrogen from CzSP18 to EtSP18.
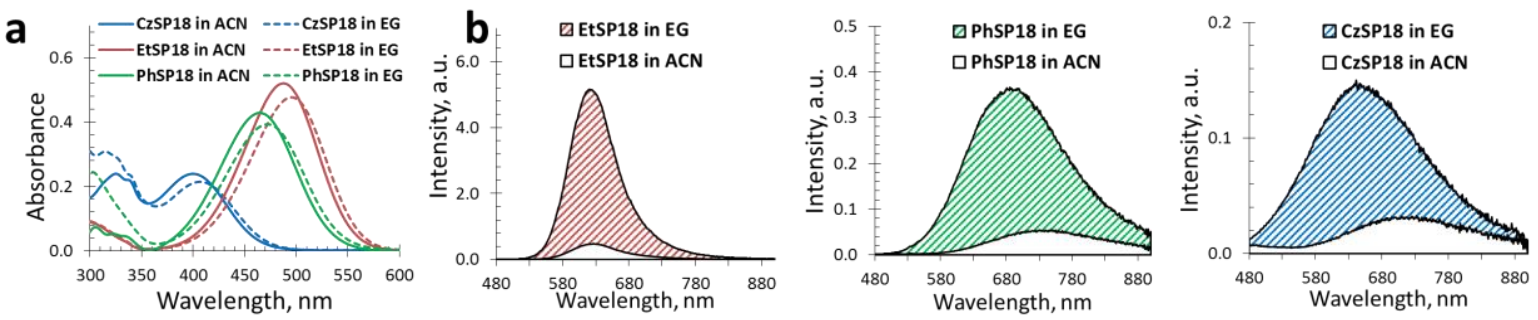

Figure 2. Spectroscopic studies of new dyes iodides in organic solvents of different viscosity. Absorption (a) and fluorescence (b) spectra of EtSP18, PhSP18 and CzSP18 in acetonitrile and ethylene glycol $\left(\lambda_{\text {exc }}=400 \pm 2 \mathrm{~nm}\right.$ for CzSP18 and $460 \pm 2 \mathrm{~nm}$ for PhSP18 and EtSP18). In the fluorescence spectra, the curves were divided by absorbance values at the excitation wavelength. Concentration of dyes was 10 and $1 \mu \mathrm{M}$ for absorption and fluorescence spectroscopy, respectively. 
Table 1. Spectroscopic properties of EtSP18, PhSP18 and CzSP18 iodides in acetonitrile and ethylene glycol. $^{\mathrm{a}}$

\begin{tabular}{lcccccc} 
& Solvent & $\varepsilon, \mathrm{M}^{-1 *} \mathrm{Cm}^{-1}$ & $\lambda_{\text {abs }}, \mathrm{nm}$ & $\lambda_{\text {emis, }}$ nm & $\begin{array}{c}\text { Stokes shift, } \\
\mathrm{nm} / \mathrm{cm}^{-1}\end{array}$ & $\Phi_{\mathrm{f}}, \%$ \\
\cline { 2 - 7 } EtSP18 & ACN & 51800 & 488 & 620 & $132 / 4363$ & 2.61 \\
& $\mathrm{EG}$ & 56600 & 495 & 615 & $120 / 3942$ & 31.9 \\
\cline { 2 - 7 } PhSP18 & $\mathrm{ACN}$ & 42300 & 464 & 725 & $261 / 7759$ & 0.61 \\
& $\mathrm{EG}$ & 46200 & 472 & 680 & $208 / 6481$ & 4.6 \\
\cline { 2 - 7 } CzSP18 & $\mathrm{ACN}$ & 24700 & 399 & 710 & $311 / 10978$ & 0.47 \\
& $\mathrm{EG}$ & 27600 & 406 & 640 & $234 / 9006$ & 2.2
\end{tabular}

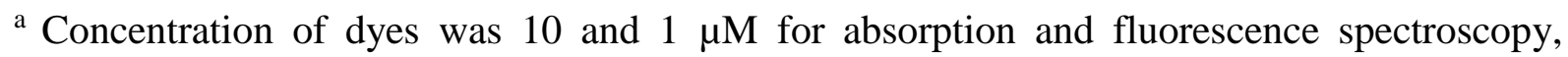
respectively.

Emission spectra showed quite different tendencies. Narrowest emission band and smallest Stokes shift were observed for EtSP18, which reflect more pronounced cyanine-like electronic configuration with delocalized positive charge in the ground state, typical for styryl pyridinium dyes. ${ }^{[6]}$ All three dyes showed poor fluorescence quantum yield (QY) in acetonitrile, whereas in more viscous glycerol a significant QY increase was observed (Fig. $2 b)$. This result is a clear indication that all three dyes are quenched by intramolecular rotation, similar to molecular rotors ${ }^{[76,77]}$ and AIE dyes. ${ }^{[26-29]}$ In both solvents, EtSP18 showed higher QY values compared to CzSP18 and PhSP18, probably because of its more pronounced cyanine-like nature. Absorption and emission spectral properties of all three dyes were independent of dye concentration (Figs. S1-S9), indicating that in this studied concentration range the effects of dye aggregation in these solvents could be neglected.

\subsection{Aggregation-induced emission of dyes in water: effect of bulky counterions}

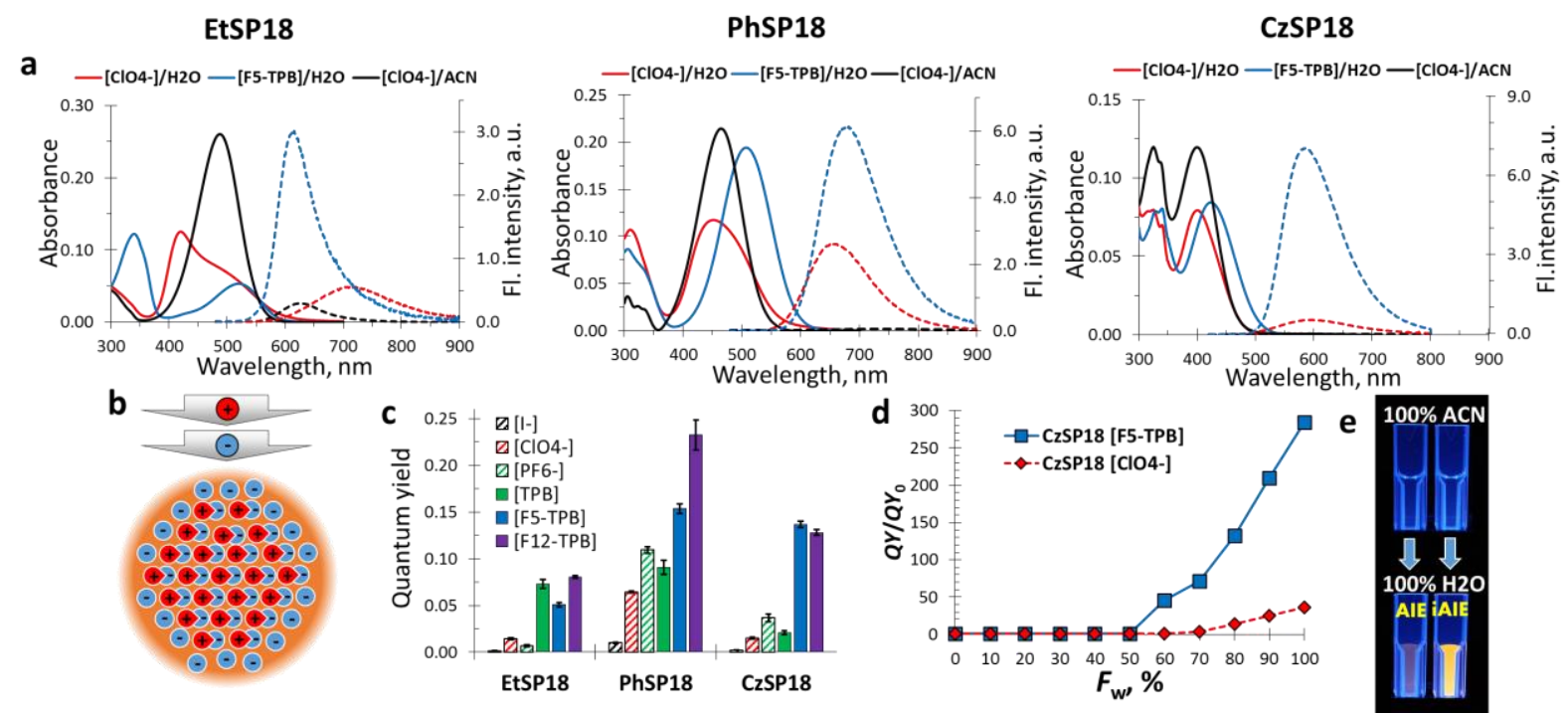

Figure 3. Neat dye salts in aqueous media. a. Absorption and fluorescence spectra of $5 \mu \mathrm{M}$ CzSP18, PhSP18 and EtSP18 in acetonitrile and NPs in water with [ClO4 $4^{-}$and [F5-TPB] counterions $\left(\lambda_{\mathrm{exc}}=400 \pm 2 \mathrm{~nm}\right.$ for CzSP18 and $460 \pm 2 \mathrm{~nm}$ for PhSP18 and EtSP18). Fluorescence spectra were divided by absorbance values at the excitation wavelength; $\mathbf{b}$. 
Schematic representation of dye NPs; c. Quantum yield of EtSP18, PhSP18 and CzSP18 NPs with different counterions; d. Fluorescence enhancement $\left(Q Y / Q Y_{0}\right)$ of $\mathbf{C z S P 1 8}$ with [ClO4 $\left.4^{-}\right]$ and [F5-TPB] in mixtures with different fractions of $\mathrm{H}_{2} \mathrm{O}$ in acetonitrile. e. Photo of cuvettes filled with $\mathbf{C z S P 1 8} /\left[\mathrm{ClO}_{4}^{-}\right]$(1) and $\mathbf{C z S P 1 8} /[\mathbf{F 5}-\mathbf{T P B}]$ (2) in acetonitrile and water.

All three dyes bear highly hydrophobic groups and therefore are expected to aggregate in water, which should enable us to study AIE properties of these dyes in the presence of different counterions. Dye aggregation was done first by diluting acetonitrile solution of dye iodides in water, followed by addition of 10 -mol excess of corresponding salts of $\left[\mathbf{I}^{-}\right],\left[\mathrm{ClO}_{4}^{-}\right]$, [PF $\left.{ }^{-}\right]$, [TPB], [F5-TPB], [F12-TPB] in order to achieve an ion exchange. This protocol was successfully used by us and others earlier to prepare fluorescent NPs. ${ }^{[52,53,78]}$ In all studied combinations of three dyes with corresponding counterions, we observed formation of aggregates in the size range $30-90 \mathrm{~nm}$, according to dynamic light scattering (Table S1). However, the dye salts of iodide, perchlorate and hexafluorophenylphosphate gave aggregates of high polydispersity and they were unstable, showing precipitation within hours/days. By contrast, aggregates of the dyes with [TPB], [F5-TPB] and [F12-TPB] gave stable nanoparticle suspensions of systematically low polydispersity $(0.1-0.2)$ with sizes $40-80 \mathrm{~nm}$ (Table S1). Previous studies suggested that bulky hydrophobic anions can provide a stabilizing effect by adsorption at the surface of newly formed nanoparticles. ${ }^{[52,57]}$

Next, we studied optical properties of aggregates of the dyes with the small inorganic anions in water. Low fluorescence signal (QY $<1 \%$ ) was observed for all $\mathrm{dye}^{+} /\left[\mathrm{I}^{-}\right]$salts probably due to iodide quenching properties (Fig. $3 \mathrm{c}$ ). In case of [ $\left.\mathrm{ClO}_{4}^{-}\right]$and $\left[\mathrm{PF}_{6}^{-}\right]$counterions, EtSP18 and CzSP18 dyes remained poorly emissive, which was not the case of PhSP18 with QY reaching $6.5 \%$ and $11 \%$ for $\left[\mathbf{C l O}_{4}^{-}\right]$and $\left[\mathbf{P F}_{6}^{-}\right]$, correspondingly. Thus, as expected, the diphenylamino propeller group rendered PhSP18 AIE properties, whereas other two SP dyes did not show pronounced AIE character. Nevertheless, fluorescence QY values for PhSP18 with inorganic counterions were quite low compared to classical AIE fluorophores, ${ }^{[26]}$ which suggested a competition between AIE and ACQ phenomena in their aggregates. In the absorption spectra, strong hypochromism was observed for the aggregated dyes in water (Fig. 3a, Figs. S10-S12). In case of EtSP18, this hypochromosim was accompanied by strong blue shift of the absorption band (Fig. 3a), indicating formation of $\mathrm{H}$-aggregates with inorganic anions, in line with the observed low QY values. For all three dyes the absorption and emission spectral properties did not change with dye concentration in the range $0.5-10 \mu \mathrm{M}$ (Figs. S13-S18), indicating that in these conditions the aggregation behavior was concentration-independent.

Very different emission properties were observed in the presence of bulky hydrophobic counterions [F5-TPB] and [F12-TPB]. Indeed, the latter drastically enhanced fluorescence intensity and QY of all three dyes in water compared to those with inorganic anions (Fig. 3). Parent non-fluorinated TPB showed intermediate behavior (Fig. 3, S10-S12): it enhanced QY of EtSP18 in water compared to inorganic anions, but it was inefficient in case of both PhSP18 and CzSP18. This result shows that in addition to the size of the counterion, fluorination also plays an important role to ensure efficient fluorescence of dyes in the aggregated state. Moreover, for all three dyes with [F5-TPB] and [F12-TPB] anions, fluorescence was much stronger compared to corresponding solutions in acetonitrile (Fig. 3a), suggesting that they behave like typical AIE fluorophores. The AIE phenomenon could be 
seen by a bare eye: when solutions of $\mathrm{CzSP18}_{[}\left[\mathrm{ClO}_{4}^{-}\right]$and $\mathrm{CzSP18} /[\mathrm{F5}-\mathrm{TPB}]$ in acetonitrile were compared to those in water, only CzSP18/[F5-TPB] showed strong emission (Fig. 3e). In acetonitrile/water solvent mixtures, CzSP18/[F5-TPB] showed strong increase in the fluorescence intensity at higher water fractions, whereas for $\mathrm{CzSP18} /\left[\mathrm{ClO}_{4}^{-}\right]$the effect was much less pronounced (Fig. 3d). These observations are remarkable because EtSP18 and CzSP18 do not exhibit pronounced AIE behavior with inorganic anions, but become typical AIEgens in the presence of bulky counterions. On the other hand, PhSP18 has intrinsic AIE properties due to propeller-shaped diphenylamino group, so that all its salts were emissive in water and the differences between small and bulky anions were much less pronounced (Fig. $3 \mathrm{c}$ and Fig. S11). Nevertheless, combination of intrinsic AIEgen properties with the effect of bulky counterion made PhSP18/[F5-TPB] and PhSP18/[F12-TPB] salts the most emissive among the studied series (Fig. 3c). This additive effect is very important for design of highly emissive materials and it is supported by our recent work, where emissive properties of an AIEgen could be further improved by using these bulky counterions. ${ }^{[50]}$ In the absorption spectra of the SP dye salts, some hypochromism was still observed compared to the acetonitrile solutions, especially for EtSP18 (Figs. S10-S12). The absorption and emission spectral properties for all three dyes with bulky counterions did not vary with dye concentration in $0.5-10 \mu \mathrm{M}$ range (as for salts with small inorganic anions), confirming that in the studied conditions the aggregation behavior was concentration-independent (Figs. S13S18). In addition, absorption bands of all three dyes with fluorinated TPBs were red shifted compared to salts with small anions without significant change in the absorption band shape (Fig. 3a, Figs. S10-S12). The latter could be related to the drop of environment polarity within the aggregates, which is expected to induce bathochromic shift in SP dyes exhibiting negative solvatochromism. ${ }^{[79]}$ Better understanding of the unique effect of bulky counterions on the optical properties of the dye salts could be achieved by X-ray analysis of eventual crystals of the dyes with [F5-TPB] and [F12-TPB]. Unfortunately, these salts were present as amorphous frozen ionic liquid-like materials, which failed to crystallize.

The observed light up of non-AIE dyes in water with assistance of bulky hydrophobic counterions can be called ionic aggregation-induced emission (iAIE). In contrast to classical AIE, this phenomenon does not require AIEgen. On the other hand, it differs from the previously described ion-association approach, which normally use highly fluorescent dyes such as rhodamine ${ }^{[57]}$ and cyanine ${ }^{[52,78]}$. Some reports showed that poorly fluorescent cationic dyes could enhance their fluorescence with addition of TPB counterions, ${ }^{[50,52]}$ but here we show that this phenomenon can be observed for both AIE and non-AIE fluorophores. However, the limitation of NPs based on neat dye salts is their tendency to dissolve in hydrophobic compartments of cells leading to dye leakage, as it was shown for rhodamine B R18 salts with [F5-TPB] and [F12-TPB] counterions. ${ }^{[57]}$ Therefore, of particular interest is to encapsulate them into rigid polymer matrix as it has already been realized for conventional bright fluorescent dyes. ${ }^{[15,59,61]}$ 


\subsection{Dye-loaded polymeric NPs}
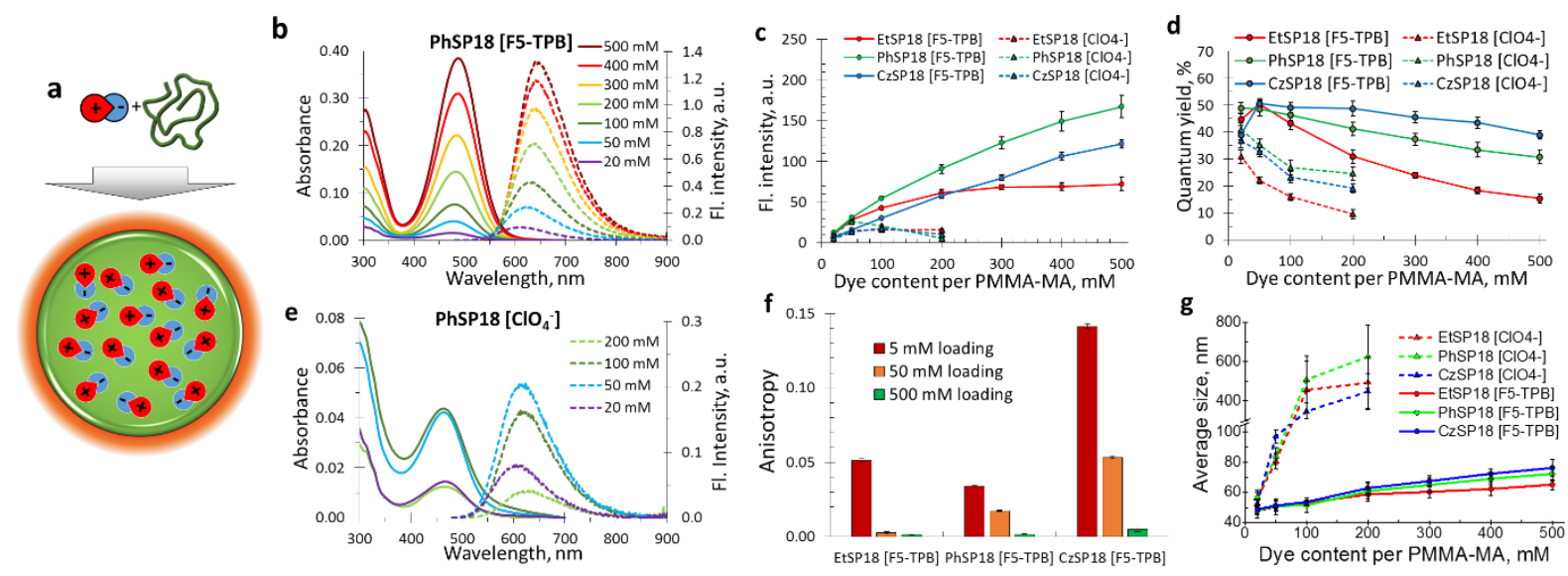

Figure 4. Dye-loaded polymeric NPs. a. Schematic representation of dye-loaded PMMA-MA NPs precipitation. b. Absorption and fluorescence spectra of PMMA-MA NPs loaded with PhSP18/[F5-TPB] at different loading. c. Integral fluorescence intensity of PMMA-MA NPs with different loading of the dyes with [F5-TPB] and [ClO4 $\left.4^{-}\right]$counterions. d. Fluorescence quantum yields of dye-loaded PMMA-MA NPs. e. Absorption and fluorescence spectra of

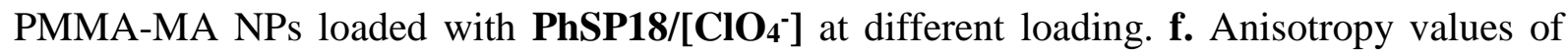
polymeric NPs loaded with $5 \mathrm{mM}, 50 \mathrm{mM}$ and $500 \mathrm{mM}$ of the dyes per PMMA-MA. g. Average size of polymeric NPs and bulky aggregates by dynamic light scattering.

Following the encouraging data with the neat dye salts, we further studied behavior of SP dye salts encapsulated into polymeric NPs. Poly(methyl methacrylate-co-methacrylic acid (PMMA-MA) NPs were formulated by charged-controlled nanoprecipitation protocol, ${ }^{[14]}$ where polymer solution in acetonitrile with different amounts of the dye was quickly diluted into phosphate buffer with vigorous stirring (Fig. 4a). Commercial PMMA-MA forms small spherical nanoparticles at $\mathrm{pH} 7.4$ stabilized with negative charge of carboxylic groups. ${ }^{[14]}$ Following well-established protocols of ion exchange, ${ }^{[60]}$ we first prepared salts of these three dyes with different counterions. Importantly, dyes with bulky hydrophobic counterions exhibited much higher mobility (Rf) on thin layer chromatography than those with small inorganic anions (Fig. S35, Table S2), indicating that the former dye salts are significantly more hydrophobic. Dyes with fluorinated TPB counterions were loaded into polymer matrix of NPs by simply adding them to acetonitrile solution before the nanoprecipitation. A wide range of dye loadings from $20 \mathrm{mM}$ to $500 \mathrm{mM}$ with respect to PMMA-MA polymer was tested, yielding small NPs with average size of $50-65 \mathrm{~nm}$, which increased slightly with loading, according to DLS measurements (Fig. 4g). By contrast, NPs of dyes with small inorganic anions loaded above $50 \mathrm{mM}$ gave large aggregates (Fig. $4 \mathrm{~g}$ ) that precipitated over time. Transmission electron microscopy (TEM) images of NPs at high loading of EtSP18, PhSP18, and CzSP18/[F5-TPB] (500 mM) showed homogeneous spherical NPs with average size of 52, 50 and $55 \mathrm{~nm}$, respectively. However, NPs formulated with PhSP18/[ClO4 $\left.{ }^{-}\right]$ showed aggregates already at $50 \mathrm{mM}$ and shapeless precipitate at $500 \mathrm{mM}$ loading (Fig. 5a, b). Bulky TPB counterions are crucial for effective loading of cationic dyes into polymeric NPs due to strong hydrophobic stabilization of ion pairs and shielding of dye positive charge, whereas dyes salts with small inorganic anions can dissociate and undergo ion exchange in aqueous medium. ${ }^{[60]}$ The latter leads to direct interaction of negatively-charged PMMA-MA 
with cationic dyes and neutralization of PMMA-MA negative surface charge and aggregation (Fig. 5c). ${ }^{[59]}$

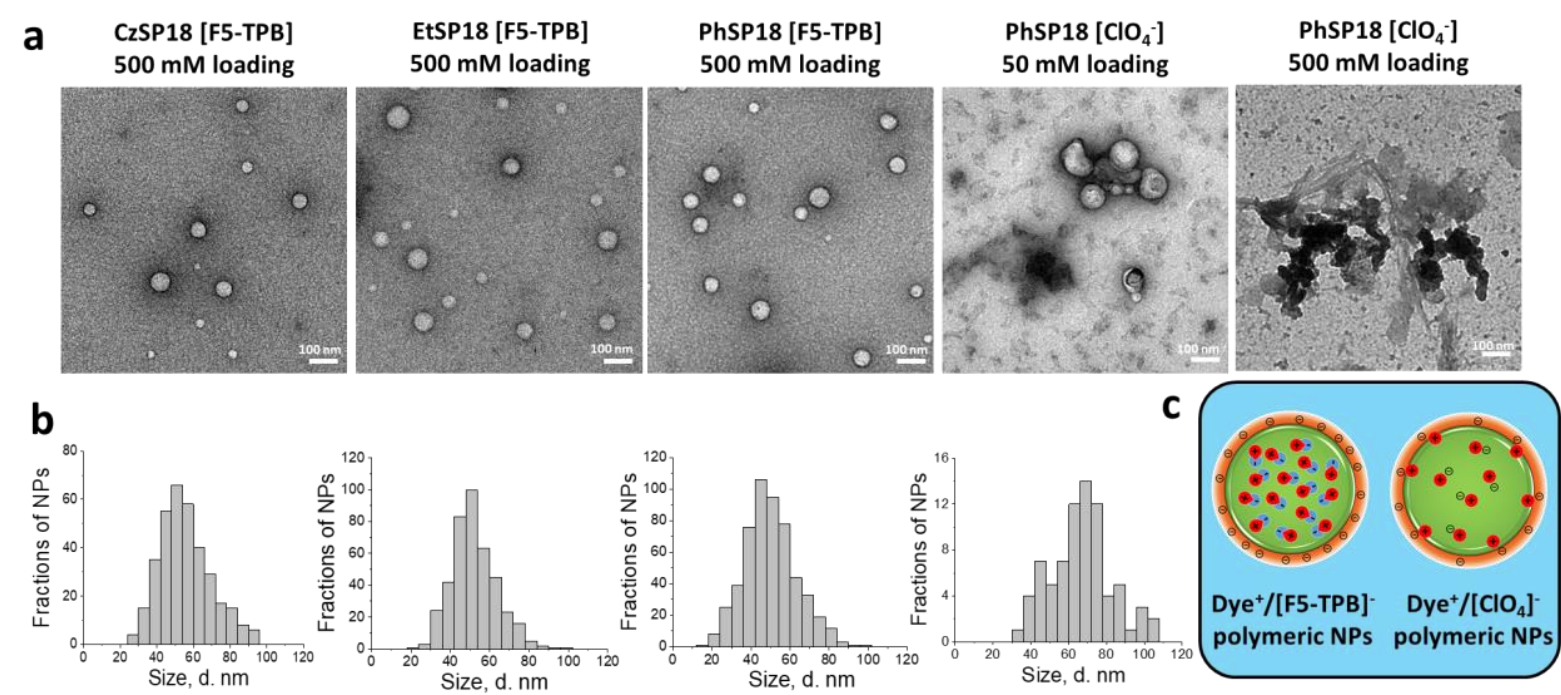

Figure 5. TEM imaging of dye-loaded polymeric NPs. a. TEM images of polymeric NPs samples loaded with $500 \mathrm{mM}$ CzSP18, EtSP18 and PhSP18 dyes with [F5-TPB] counterion;

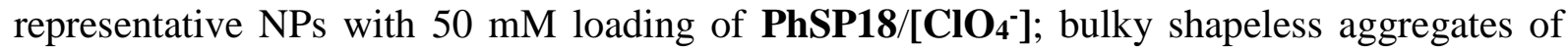

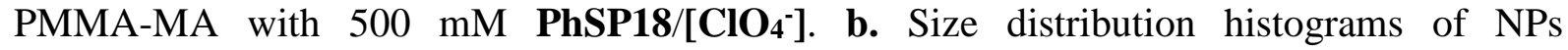
corresponding to the TEM images (top). c. Schematic presentation of NPs encapsulated with PhSP18/[F5-TPB] and PhSP18/[ClO4$\left.{ }^{-}\right]$.

Light absorption of NPs increased gradually with dye loading in the range from $20 \mathrm{mM}$ to 500 $\mathrm{mM}$ for the ion pairs with bulky counterions (Fig. 4b and Figs. S4-S18). By contrast, for small anions, saturation of absorbance was observed already at $50 \mathrm{mM}$, followed by further drop of absorbance at $200 \mathrm{mM}$, which is probably related to particle aggregation and further precipitation (Fig. 4e and Figs. S4-S18). Fluorescence signal also increased with dye loading for the three dyes with bulky counterions, whereas for small anions rapid signal saturation was observed already at $50 \mathrm{mM}$ loading, followed by strong drop at $200 \mathrm{mM}$ (Fig. 4d). Remarkably, fluorescence quantum yields for PhSP18 and CzSP18 NPs with bulky counterions were very high (30-50\%), with only a small decrease at the highest $(500 \mathrm{mM})$ loading to $30 \%$ and $40 \%$, respectively. The high QY values were observed for both [F5-TPB] and [F12-TPB] anions, whereas for small inorganic anions they were significantly lower even for $100 \mathrm{mM}$ loading (Figs. S19-S21). Thus, bulky counterions seem to play a crucial role in lighting up dyes and preventing their ACQ inside polymeric NPs. Importantly, they enhanced emission for both AIEgen PhSP18 and dyes without AIE character (EtSP18 and CzSP18). In pure salts and in polymer NPs with small anions, PhSP18 show systematically higher QYs values (Figs. 3c and 4d), which is probably because of intrinsic AIE property of the dye. However, when bulky counterions are used in polymeric NPs, a non-AIE dye CzSP18 is most emissive (Fig. 4d). This indicates that bulky counterion can light up a poorly emissive dye inside a polymer matrix independently of the AIE character of the dye. In fact, the intrinsic AIE property of the dye does not play important role when it is encapsulated into the hydrophobic matrix, because polymer interferes with dye aggregation and favors uniform distribution in the matrix, as it was shown previously for other AIE dyes. ${ }^{[48]}$ On the other hand, counterion seems to overcome this limitation as by itself it can assemble the dyes inside the polymer minimizing the ACQ. ${ }^{[59,60]}$ To confirm whether the dyes are indeed assembled 
inside polymeric NPs in a close proximity we made fluorescence anisotropy measurements. This method enables direct access to extent of dye-dye excitation energy migration, which reflects qualitatively the dye-dye proximity. ${ }^{[63,80]}$ It was found that increase in the dye loading for all three dyes with [F5-TPB] counterion led to a drop in the fluorescence anisotropy reaching nearly zero values at $500 \mathrm{mM}$, indicating a drastic decrease in the dye-dye spacing. It should be noted that the anisotropy loss is usually observed for dye assemblies with small Stokes shift $\left(21 \mathrm{~nm} / 686 \mathrm{~cm}^{-1}\right.$ for rhodamine B in ethanol), where homo-FRET process can take place easily. ${ }^{[23,63]}$ In the present case, it takes place for dyes with much larger Stokes shift (Table 1) and thus poorer spectral overlap for homo-FRET. Therefore, observation of nearlyzero anisotropy and high QY values at $500 \mathrm{mM}$ loading suggests that the dyes are probably aggregated inside the polymer matrix, but the ACQ is blocked by the counterion, preventing the dyes from direct pi-stacking. Among the three tested dyes, the loss of anisotropy was fastest in case of EtSP18, which is probably because it has the smallest Stokes shift and thus can easier undergo energy hopping through homo-FRET processes than the other dyes.

These studies suggest that iAIE phenomenon can be also observed inside polymeric matrixes and thus can be directly applied for preparation of highly fluorescent polymeric NPs. To the best of our knowledge, it is the first report where the counterion approach was successfully used in combination with poorly emissive non-AIE fluorophores to prepare highly emissive dye-loaded polymeric NPs.

\subsection{Single-particle fluorescence}

Next, we examined the developed NPs by single-particle imaging using wide-field fluorescence microscopy. The new NPs were compared with commercial Quantum dots 605 (Qdots 605), presenting emission maximum in similar wavelength range. First, we prepared polymeric NPs loaded with $5 \mathrm{mM}, 50 \mathrm{mM}$ and $500 \mathrm{mM}$ loading of PhSP18/[F5-TPB] to quantify single-particle brightness and its distribution (Fig. 6a). After 1000-times dilution of polymeric NPs and Qdots 605 with a phosphate buffer, they were immobilized at the polyethyleneimine-covered glass surface and studied by fluorescence microscopy. Singleparticle brightness increased gradually with the dye loading in polymeric NPs (Fig. 6b). Images of NPs with lowest PhSP18/[F5-TPB] concentration (5 mM) showed less fluorescent spots, which basically could be explained by presence of empty polymeric NPs without fluorescent dyes. Image analysis revealed that NPs encapsulating $500 \mathrm{mM}$ PhSP18/[F5-TPB] were 50-fold brighter than Qdots 605 at $470 \mathrm{~nm}$ excitation wavelength, optimal for both NPs (Fig. 6c). We should note here that this comparison is affected by significantly larger size of our particles compared to Qdots 605 ( $\sim 50 \mathrm{~nm}$ vs $\sim 25 \mathrm{~nm})$. On the other hand, the advantage of our system compared to Qdots is that the size of fluorescent core in our NPs can be tuned to much larger extent within $9-100 \mathrm{~nm}^{[14,15]}$ 

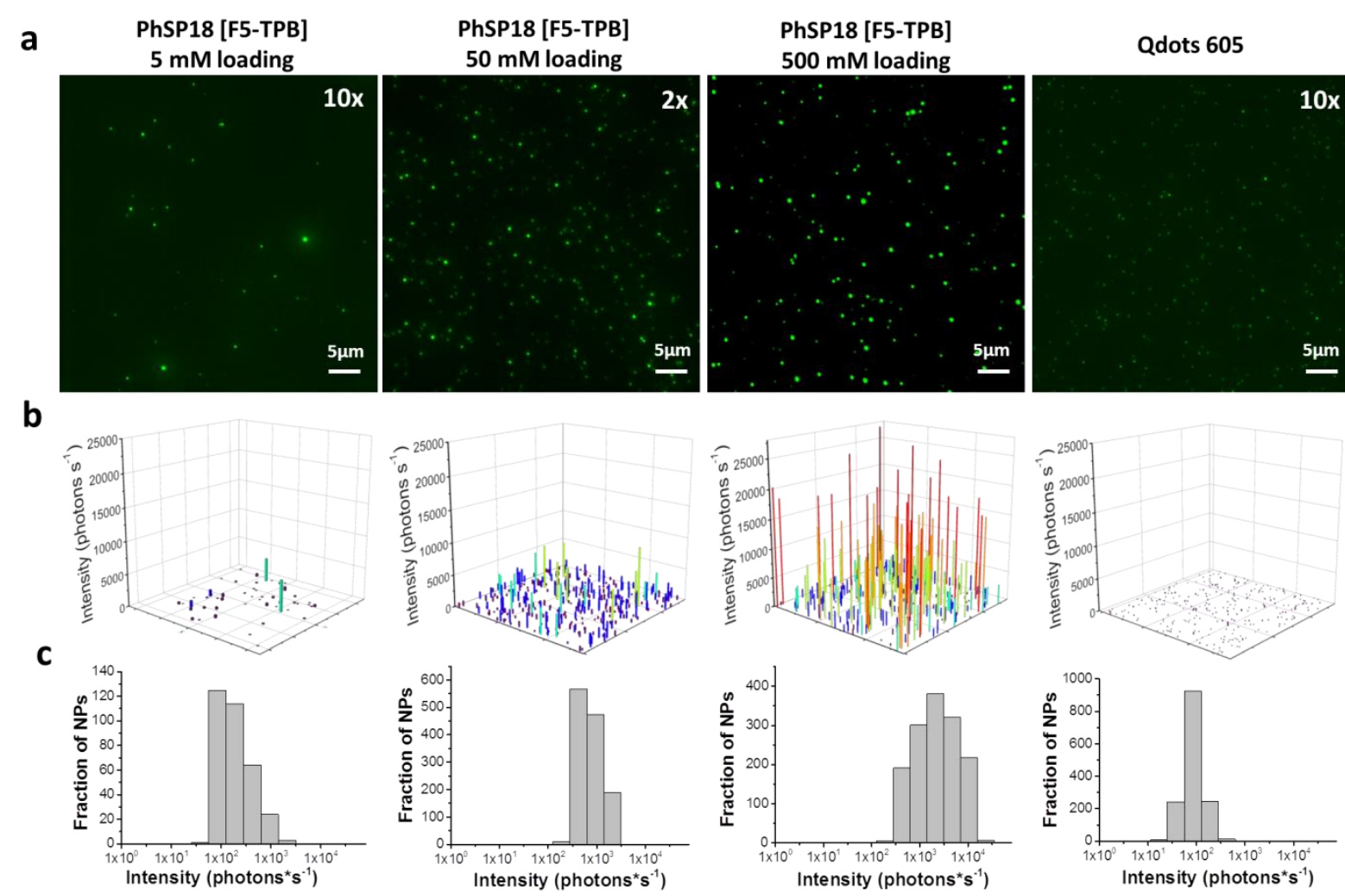

Figure 6. Single-particle characterization of NPs at different loading of PhSP18/[F5-TPB] and reference quantum dots 605 (Qdots 605) by wide-field fluorescence microscopy. a. Single-particle fluorescence images of polymeric NPs loaded with $5 \mathrm{mM}, 50 \mathrm{mM}$ and 500 $\mathrm{mM}$ PhSP18/[F5-TPB] and Qdots 605. For visibility, images of Qdots 605 and polymeric NPs with $5 \mathrm{mM}$ PhSP18 are presented with 10-fold intensity amplification and NPs with 50 $\mathrm{mM}$ loading - with 2-fold amplification. b. 3D brightness histograms referred to the corresponding fluorescence images (top). c. Corresponding distribution histograms of NPs brightness. Wide-field fluorescence microscopy was performed with excitation at $470 \mathrm{~nm}$ (power density of $0.51 \mathrm{~W} \mathrm{~cm}^{-2}$ ); emission was collected at $>500 \mathrm{~nm}$ with a long-pass filter.

We also compared single-particle fluorescence of NPs loaded with EtSP18, PhSP18 and CzSP18 (Fig. 7a). In line with spectroscopy data in cuvette, PhSP18 NPs demonstrated the highest brightness per particle (Fig. 7b). Video-rate imaging of NPs revealed single-particle traces with good photostability (Fig. 7c) and absence of blinking (Fig. 7d). Interestingly, highest photostability upon irradiation was observed for CzSP18 NPs, which revealed intermediate fluorescence brightness (Fig. 7c). Absence of blinking is an advantage of the newly developed particles compared to Qdots, which showed expected whole particle blinking (Fig. 7e). It is also advantageous compared to our brightest dye-loaded polymeric NPs based on rhodamine (R18) and [F5-TPB], which showed intermittent or complete blinking process. ${ }^{[63]}$ The stable emission of the newly developed NPs is probably related to much larger Stokes shift of this dye, which slows down the energy migration. Even though the energy migration takes place in these NPs, according to fluorescence anisotropy data, it is probably much slower, thus decreasing the dye cooperativity and thus the whole-particle blinking. ${ }^{[4,59,60,63]}$ 

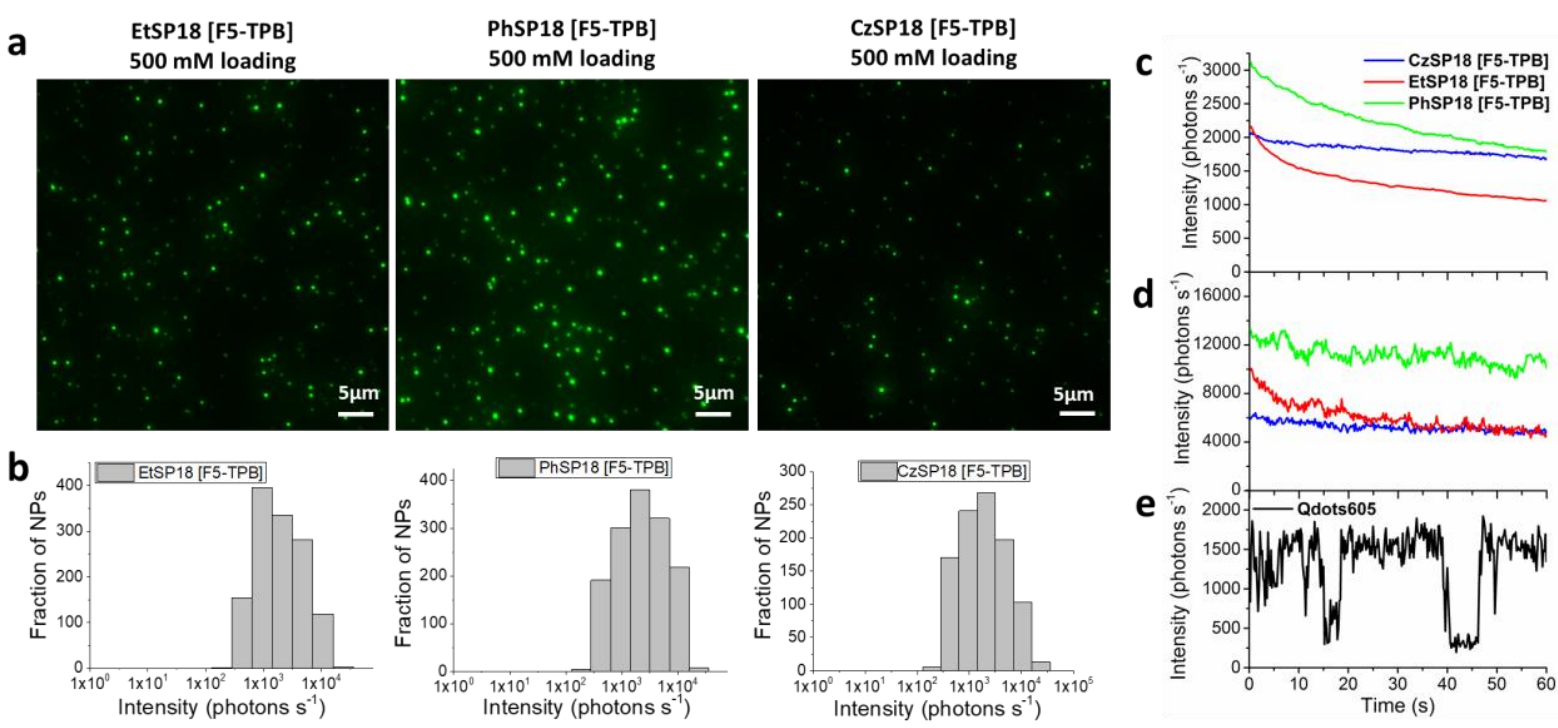

Figure 7. Single-particle characterization of polymeric NPs loaded with $500 \mathrm{mM}$ EtSP18/[F5-TPB], PhSP18/[F5-TPB] and CzSP18/[F5-TPB]. a. Single-particle fluorescence images of dye-loaded NPs. b. Corresponding distribution histograms of NPs brightness. c. Photobleaching profiles of polymeric NPs loaded with $500 \mathrm{mM}$ CzSP18, EtSP18 and PhSP18 dyes with [F5-TPB] counterion. d. Single-particle traces of the NPs. e. Single-particle trace of Qdots 605.

\section{Conclusion}

We report here an "ionic" aggregation-induced emission (iAIE) promoted by formation of stable ion pairs of poorly fluorescent non-AIE dyes and their application for preparation of bright dye-loaded polymeric nanoparticles. Three hydrophobic derivatives of styryl pyridinium dyes have been developed: two planar fluorophores (EtSP18 and CzSP18), bearing diethylamino and carbazolyl moieties (non-AIE dyes), and propeller shaped dye PhSP18 with diphenylamino group (AIE dye). The dyes showed poor fluorescence in organic solutions, but enhance their emission in viscous media preventing rotational quenching. We found that bulky fluorinated tetraphenylborate counterions [F5-TPB] and [F12-TPB] light up fluorescence of all three dyes in water, whereas with small inorganic anions $\left[\mathbf{P F}_{6}{ }^{-}\right]$and $\left[\mathbf{C l O}_{4}{ }^{-}\right.$ ] only AIE dye PhSP18 shows some fluorescence enhancement. Thus, we observe "ionic" AIE phenomenon that can operate with intrinsically non-AIE charged dyes. Fluorescence QY of pure PhSP18 (AIE) and CzSP18 (non-AIE) salts with bulky counterions reaches $23 \%$ and $14 \%$, respectively, suggesting that the counterion-based iAIE and AIE phenomena are additive. We applied our findings to preparation of dye-loaded polymeric NPs based on biocompatible PMMA-MA. For all three dyes, bulky counterions enable preparation of bright dye-loaded polymeric NPs, featuring $\sim 50 \mathrm{~nm}$ size and good fluorescence quantum yields reaching $40 \%$ at $500 \mathrm{mM}$ dye loading. The quantum yield increased in the following order: EtSP18 < PhSP18 < CzSP18, indicating that inside polymeric NPs it is independent of the intrinsic AIE character of dyes, but controlled by the bulky counterions. In sharp contrast, attempts to encapsulate the dye salts with small inorganic anions $\left[\mathbf{I}^{-}\right],\left[\mathbf{C l O}_{4}^{-}\right]$and $\left[\mathbf{P F}_{\mathbf{6}}^{-}\right]$into 
polymeric NPs gave large aggregates with low quantum yield at high dye loading. Thus, thanks to iAIE with bulky counterions, for the first time polymeric NPs loaded with poorly fluorescent dyes reach $40 \%$ quantum yield at $500 \mathrm{mM}$ dye loading, rendering NPs high brightness. Single-particle measurements revealed that polymeric NPs loaded with PhSP18 with [F5-TPB] counterions are 50-fold brighter than quantum dots Qdots 605, excited at 470 $\mathrm{nm}$. The new NPs show stable non-blinking fluorescent signal, which is an additional advantage compared to QDots. Another interesting feature of these NPs is their large Stokes shift $(\sim 200 \mathrm{~nm})$, which is useful to minimize background noise due to scattering and autofluorescence.

Thus, the described iAIE phenomenon show that weakly emissive dyes presenting or not AIE characteristics can light up in the aggregated form in water or polymeric matrix thanks to bulky hydrophobic counterions. As the studied dyes present molecular rotor properties, they turn on their emission probably because of restriction of intramolecular rotation (RIR) in the aggregated form, as it was reported earlier for AIE dyes. ${ }^{[26-28]}$ However, bulky hydrophobic counterions can light up also non-AIE molecular rotor dyes with flat architecture (e.g. CzSP18) probably because they serve as a spacer between the dyes in the aggregated state, as it was observed for highly emissive rhodamine and cyanine dyes. ${ }^{[57,59,60]}$ Comparative studies revealed that counterion fluorination is also very important for efficient emission in the aggregated form, which is probably related to (i) negative charge delocalization among large number of fluorines ${ }^{[81,82]}$ and (ii) super-hydrophobicity of fluorinated compounds. ${ }^{[83]}$ High hydrophobicity and delocalized charge of the anion prevents its solvation and dissociation of the dye salts in water. Therefore, in contrast to highly hydrated inorganic anions that allow flat cationic dyes to pi-stack in water, poorly solvated bulky hydrophobic anions interact strongly with cationic dyes through electrostatic and hydrophobic forces, thus preventing them from pi-stacking. Moreover, high hydrophobicity of these counterions ensures efficient encapsulation of cationic dyes inside hydrophobic matrix of polymeric NPs, preserving their strong emission, in line with our previous works on highly emissive fluorophores based on rhodamine and cyanines. ${ }^{[60-62]}$

Overall, the described iAIE phenomenon provides a bridge between AIE and bulky hydrophobic counterions approaches to fight ACQ. On one hand, iAIE extends the counterion concept to large variety of ionic dyes presenting weak fluorescence. On the other hand, it shows the way to generate bright AIE materials based on non-AIE fluorophores using specially selected bulky counterions.

\section{Experimental Section}

Materials and methods, synthesis of the styryl pyridinium dyes and their ion-exchange with different anions, the details of nanoparticle preparation, single-particle fluorescence measurements and TEM measurements are described in the Supporting Information.

\section{Supporting Information}

Supporting Information is available from the Wiley Online Library or from the author. 


\section{Acknowledgements}

I.O.A. acknowledges Marie Curie post-doctoral research grant (H2020-MSCA-IF-2018, DNANanoProbes 846571) by the European Union. This work was supported by the European Research Council ERC Consolidator grant BrightSens 648525. The authors also thank C. Crucifix from the FRISBI platform for help with electron microscopy.

\section{Conflict of Interest}

The authors declare no conflict of interest.

\section{References}

[1] O. S. Wolfbeis, Chem. Soc. Rev. 2015, 44, 4743.

[2] P. D. Howes, R. Chandrawati, M. M. Stevens, Science 2014, 346, 1247390.

[3] K. Li, B. Liu, Chem. Soc. Rev. 2014, 43, 6570.

[4] A. Reisch, A. S. Klymchenko, Small 2016, 12, 1968.

[5] W. C. W. Chan, D. J. Maxwell, X. Gao, R. E. Bailey, M. Han, S. Nie, Curr. Opin. Biotechnol. 2002, 13, 40.

[6] I. L. Medintz, H. T. Uyeda, E. R. Goldman, H. Mattoussi, Nat. Mater. 2005, 4, 435.

[7] X. Michalet, F. F. Pinaud, L. A. Bentolila, J. M. Tsay, S. Doose, J. J. Li, G. Sundaresan, A. M. Wu, S. S. Gambhir, S. Weiss, Science 2005, 307, 538.

[8] M. Montalti, L. Prodi, E. Rampazzo, N. Zaccheroni, Chem. Soc. Rev. 2014, 43, 4243.

[9] S. W. Bae, W. Tan, J. I. Hong, Chem. Commun. 2012, 48, 2270.

[10] V. N. Mochalin, O. Shenderova, D. Ho, Y. Gogotsi, Nat. Nanotechnol. 2012, 7, 11.

[11] S.-T. Yang, L. Cao, P. G. Luo, F. Lu, X. Wang, H. Wang, M. J. Meziani, Y. Liu, G. Qi, Y.-P. Sun, J. Am. Chem. Soc. 2009, 131, 11308.

[12] H. S. Peng, D. T. Chiu, Chem. Soc. Rev. 2015, 44, 4699.

[13] C. B. Winiger, S. Li, G. R. Kumar, S. M. Langenegger, R. Häner, Angew. Chemie - Int. Ed. 2014, 53, 13609.

[14] A. Reisch, A. Runser, Y. Arntz, Y. Mély, A. S. Klymchenko, ACS Nano 2015, 9, 5104.

[15] A. Reisch, D. Heimburger, P. Ernst, A. Runser, P. Didier, D. Dujardin, A. S. Klymchenko, Adv. Funct. Mater. 2018, 28, 1805157.

[16] F. Würthner, T. E. Kaiser, C. R. Saha-Möller, Angew. Chemie - Int. Ed. 2011, 50, 3376.

[17] A. Okamoto, Chem. Soc. Rev. 2011, 40, 5815.

[18] V. M. Farzan, M. V. Kvach, I. O. Aparin, D. E. Kireev, T. A. Prikazchikova, A. V. Ustinov, V. V. Shmanai, G. A. Shipulin, V. A. Korshun, T. S. Zatsepin, Talanta 2019, 194, 226.

[19] F. Bouhedda, K. T. Fam, M. Collot, A. Autour, S. Marzi, A. Klymchenko, M. Ryckelynck, Nat. Chem. Biol. 2020, 16, 69.

[20] X. Zhang, Z. Chen, F. Würthner, J. Am. Chem. Soc. 2007, 129, 4886.

[21] Z. Tian, A. D. Shaller, A. D. Q. Li, Chem. Commun. 2009, 180.

[22] C. Grazon, J. Rieger, R. Méallet-Renault, B. Charleux, G. Clavier, Macromolecules 2013, 46, 5167.

[23] K. Trofymchuk, A. Reisch, I. Shulov, Y. Mély, A. S. Klymchenko, Nanoscale 2014, 6, 12934.

[24] B. Zhang, H. Soleimaninejad, D. J. Jones, J. M. White, K. P. Ghiggino, T. A. Smith, W. W. H. Wong, Chem. Mater. 2017, 29, 8395. 
[25] J. Luo, Z. Xie, Z. Xie, J. W. Y. Lam, L. Cheng, H. Chen, C. Qiu, H. S. Kwok, X. Zhan, Y. Liu, D. Zhu, B. Z. Tang, Chem. Commun. 2001, 18, 1740.

[26] J. Mei, N. L. C. Leung, R. T. K. Kwok, J. W. Y. Lam, B. Z. Tang, Chem. Rev. 2015, $115,11718$.

[27] J. Qian, B. Z. Tang, Chem 2017, 3, 56.

[28] Y. Chen, J. W. Y. Lam, R. T. K. Kwok, B. Liu, B. Z. Tang, Mater. Horizons 2019, 6, 428.

[29] Y. Hong, J. W. Y. Lam, B. Z. Tang, Chem. Soc. Rev. 2011, 40, 5361.

[30] Z. R. Grabowski, K. Rotkiewicz, W. Rettig, Chem. Rev. 2003, 103, 3899.

[31] J. Huang, H. Nie, J. Zeng, Z. Zhuang, S. Gan, Y. Cai, J. Guo, S. J. Su, Z. Zhao, B. Z. Tang, Angew. Chemie - Int. Ed. 2017, 56, 12971.

[32] D. Zhao, F. Fan, J. Cheng, Y. Zhang, K. S. Wong, V. G. Chigrinov, H. S. Kwok, L. Guo, B. Z. Tang, Adv. Opt. Mater. 2015, 3, 199.

[33] S. Guo, Y. Song, Y. He, X. Y. Hu, L. Wang, Angew. Chemie - Int. Ed. 2018, 57, 3163.

[34] L. Xu, Z. Wang, R. Wang, L. Wang, X. He, H. Jiang, H. Tang, D. Cao, B. Z. Tang, Angew. Chemie - Int. Ed. 2019, DOI: 10.1002/anie.201907678.

[35] J. Qi, C. Sun, A. Zebibula, H. Zhang, R. T. K. K. Kwok, X. Zhao, W. Xi, J. W. Y. Y. Lam, J. Qian, B. Z. Tang, Adv. Mater. 2018, 30, 1706856.

[36] P. Wei, J. X. Zhang, Z. Zhao, Y. Chen, X. He, M. Chen, J. Gong, H. H. Y. Sung, I. D. Williams, J. W. Y. Lam, B. Z. Tang, J. Am. Chem. Soc. 2018, 140, 1966.

[37] F. De Nisi, R. Francischello, A. Battisti, A. Panniello, E. Fanizza, M. Striccoli, X. Gu, N. L. C. Leung, B. Z. Tang, A. Pucci, Mater. Chem. Front. 2017, 1, 1406.

[38] M. Gao, B. Z. Tang, ACS Sensors 2017, 2, 1382.

[39] W. Qin, D. Ding, J. Liu, W. Z. Yuan, Y. Hu, B. Liu, B. Z. Tang, Adv. Funct. Mater. 2012, 22, 771 .

[40] N. Alifu, A. Zebibula, J. Qi, H. Zhang, C. Sun, X. Yu, D. Xue, J. W. Y. Lam, G. Li, J. Qian, B. Z. Tang, ACS Nano 2018, 12, 11282.

[41] L. Zong, H. Zhang, Y. Li, Y. Gong, D. Li, J. Wang, Z. Wang, Y. Xie, M. Han, Q. Peng, X. Li, J. Dong, J. Qian, Q. Li, Z. Li, ACS Nano 2018, 12, 9532.

[42] Z. Zhu, J. Qian, X. Zhao, W. Qin, R. Hu, H. Zhang, D. Li, Z. Xu, B. Z. Tang, S. He, ACS Nano 2016, 10, 588 .

[43] X. Zhang, K. Wang, M. Liu, X. Zhang, L. Tao, Y. Chen, Y. Wei, Nanoscale 2015, 7, 11486.

[44] X. Zhang, X. Zhang, B. Yang, M. Liu, W. Liu, Y. Chen, Y. Wei, Polym. Chem. 2014, $5,399$.

[45] G. Feng, B. Liu, Acc. Chem. Res. 2018, 51, 1404.

[46] K. Li, Z. Zhu, P. Cai, R. Liu, N. Tomczak, D. Ding, J. Liu, W. Qin, Z. Zhao, Y. Hu, X. Chen, B. Z. Tang, B. Liu, Chem. Mater. 2013, 25, 4181.

[47] D. Ding, C. C. Goh, G. Feng, Z. Zhao, J. Liu, R. Liu, N. Tomczak, J. Geng, B. Z. Tang, L. G. Ng, B. Liu, Adv. Mater. 2013, 25, 6083.

[48] J. Geng, K. Li, W. Qin, L. Ma, G. G. Gurzadyan, B. Z. Tang, B. Liu, Small 2013, 9, 2012.

[49] X. Gu, X. Zhang, H. Ma, S. Jia, P. Zhang, Y. Zhao, Q. Liu, J. Wang, X. Zheng, J. W. Y. Lam, D. Ding, B. Z. Tang, Adv. Mater. 2018, 30, 1801065.

[50] N. Adarsh, A. S. Klymchenko, Nanoscale 2019, 11, 13977.

[51] L. D. Lavis, R. T. Raines, ACS Chem. Biol. 2014, 9, 855.

[52] H. Yao, M. Yamashita, K. Kimura, Langmuir 2009, 25, 1131.

[53] H. Yao, K. Ashiba, RSC Adv. 2011, 1, 834.

[54] D. K. Bwambok, B. El-Zahab, S. K. Challa, M. Li, L. Chandler, G. A. Baker, I. M. Warner, ACS Nano 2009, 3, 3854. 
[55] A. N. Jordan, S. Das, N. Siraj, S. L. De Rooy, M. Li, B. El-Zahab, L. Chandler, G. A. Baker, I. M. Warner, Nanoscale 2012, 4, 5031.

[56] J.-F. Lamère, N. Saffon, I. Dos Santos, S. Fery-Forgues, Langmuir 2010, 26, 10210.

[57] I. Shulov, S. Oncul, A. Reisch, Y. Arntz, M. Collot, Y. Mely, A. S. Klymchenko, Nanoscale 2015, 7, 18198.

[58] P. K. S. Magut, S. Das, V. E. Fernand, J. Losso, K. McDonough, B. M. Naylor, S. Aggarwal, I. M. Warner, J. Am. Chem. Soc. 2013, 135, 15873.

[59] A. Reisch, P. Didier, L. Richert, S. Oncul, Y. Arntz, Y. Mély, A. S. Klymchenko, Nat. Commun. 2014, 5, 4089.

[60] B. Andreiuk, A. Reisch, E. Bernhardt, A. S. Klymchenko, Chem. - An Asian J. 2019, $14,836$.

[61] B. Andreiuk, A. Reisch, M. Lindecker, G. Follain, N. Peyriéras, J. G. Goetz, A. S. Klymchenko, Small 2017, 13, 1.

[62] B. Andreiuk, A. Reisch, V. G. Pivovarenko, A. S. Klymchenko, Mater. Chem. Front. 2017, 1, 2309.

[63] A. Reisch, K. Trofymchuk, A. Runser, G. Fleith, M. Rawiso, A. S. Klymchenko, ACS Appl. Mater. Interfaces 2017, 9, 43030.

[64] K. Trofymchuk, A. Reisch, P. Didier, F. Fras, P. Gilliot, Y. Mely, A. S. Klymchenko, Nat. Photonics 2017, 11, 657.

[65] N. Melnychuk, A. S. Klymchenko, J. Am. Chem. Soc. 2018, 140, 10856.

[66] T. Deligeorgiev, A. Vasilev, S. Kaloyanova, J. J. Vaquero, Color. Technol. 2010, 126, 55.

[67] R. Krieg, A. Eitner, W. Günther, C. Schürer, J. Lindenau, K. J. Halbhuber, J. Mol. Histol. 2008, 39, 169.

[68] P. Yan, A. Xie, M. Wei, L. M. Loew, J. Org. Chem. 2008, 73, 6587.

[69] P. Yan, C. D. Acker, W. L. Zhou, P. Lee, C. Bollensdorff, A. Negreane, J. Lotti, L. Sacconi, S. D. Antic, P. Kohl, H. D. Mansvelder, F. S. Pavone, L. M. Loew, Proc. Natl. Acad. Sci. U. S. A. 2012, 109, 20443.

[70] S. Bolte, C. Talbot, Y. Boutte, O. Catrice, N. D. Read, B. Satiat-Jeunemaitre, J. Microsc. 2004, 214, 159.

[71] M. A. Gaffield, W. J. Betz, Nat. Protoc. 2007, 1, 2916.

[72] J. Yu, Y. Cui, H. Xu, Y. Yang, Z. Wang, B. Chen, G. Qian, Nat. Commun. 2013, 4, 2719.

[73] X. Liu, H. Gu, M. Wang, X. Du, B. Gao, A. Elbaz, L. Sun, J. Liao, P. Xiao, Z. Gu, Adv. Mater. 2018, 30, 1800103.

[74] K. Trofymchuk, J. Valanciunaite, B. Andreiuk, A. Reisch, M. Collot, A. S. Klymchenko, J. Mater. Chem. B 2019, 7, 5199.

[75] B. Jedrzejewska, A. Rudnicki, Dye. Pigment. 2009, 80, 297.

[76] M. A. Haidekker, M. Nipper, A. Mustafic, D. Lichlyter, M. Dakanali, E. A. Theodorakis, in Adv. Fluoresc. Reporters Chem. Biol. I, Springer, Berlin, Heidelberg, 2010, pp. 267-308.

[77] M. K. Kuimova, Phys. Chem. Chem. Phys. 2012, 14, 12671.

[78] S. Das, D. Bwambok, B. El-Zahab, J. Monk, S. L. De Rooy, S. Challa, M. Li, F. R. Hung, G. A. Baker, I. M. Warner, Langmuir 2010, 26, 12867.

[79] D. Sahoo, P. Bhattacharya, S. Chakravorti, J. Phys. Chem. B 2011, 115, 10983.

[80] R. Camacho, D. Täuber, I. G. Scheblykin, Adv. Mater. 2019, 31, 1805671.

[81] I. Krossing, I. Raabe, Angew. Chemie - Int. Ed. 2004, 43, 2066.

[82] S. H. Strauss, Chem. Rev. 1993, 93, 927.

[83] M. Cametti, B. Crousse, P. Metrangolo, R. Milani, G. Resnati, Chem. Soc. Rev. 2012, $41,31$. 
[84] M. La Deda, M. Ghedini, I. Aiello, T. Pugliese, F. Barigelletti, G. Accorsi, J. Organomet. Chem. 2005, 690, 857.

\section{The table of contents entry}

Keyword: ionic AIE

Ilya O. Aparin, Nina Melnychuk, and Andrey S. Klymchenko*

Ionic aggregation-induced emission: bulky counterions light up dyes in polymeric nanoparticles

A phenomenon of ionic aggregation-induced emission (iAIE) is described, which combines concepts of AIE and bulky hydrophobic counterions to fight aggregation-caused quenching in cationic weakly emissive fluorophores. iAIE has been applied for preparation of bright dyeloaded polymeric nanoparticles exhibiting $\sim 50$-fold stronger emission without blinking compared to quantum dots 605 in single-particle microscopy.

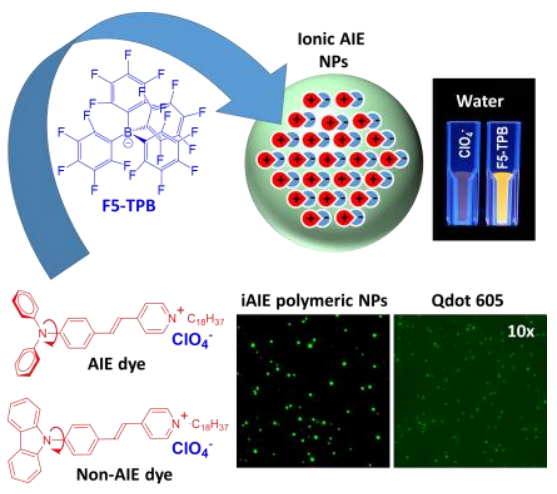

